\title{
Jaundice and Pregnancy: Why Going Viral Is Out of Style
}

\author{
Erica R. Cohen ${ }^{1}$ Tram T. Tran ${ }^{2}$
}

Published online: 23 November 2016

(c) Springer Science+Business Media New York 2016

Management of the pregnant woman with abnormal liver tests is a common, albeit challenging, clinical endeavor. A multidisciplinary collaboration between obstetrician and gastroenterologist is required in considering both expectant mother and fetus in management decisions. Diagnosing pregnancy-related causes of abnormal liver function tests is important as urgent obstetric intervention and delivery may be needed. Conversely, conditions not related to pregnancy might be exacerbated by fetal delivery. The spectrum of symptoms caused by abnormal liver tests ranges from mild illness to significant risk of morbidity and mortality to the mother and fetus. Understanding the prevalence of each possible etiology of abnormal liver tests in the pregnant patient is critical to appropriate and timely clinical management.

The prevalence of abnormal liver function tests among expectant mothers is $0.3-3 \%$ [1]. For 70 years, acute viral hepatitis was thought to be the leading cause worldwide, based on epidemiologic studies conducted primarily outside of the USA [2,3], although one US study also reported that hepatitis B accounted for $40 \%$ of expectant mothers with symptoms and signs of acute hepatitis [4]. Importantly, these landmark studies were conducted prior to 1991, after which widespread hepatitis vaccination was

Erica R. Cohen

Erica.Cohen@cshs.org

Tram T. Tran

Tram.Tran@cshs.org

1 Division of Digestive and Liver Diseases, Cedars Sinai Medical Center, 8730 Alden Dr, Thalians Bldg, Los Angeles, CA 90048, USA

2 Liver Transplant, Cedars Sinai Medical Center, 8900 Beverly Boulevard, Los Angeles, CA 90048, USA implemented. Recently, geographically diverse studies have revisited the epidemiologic dogma informing the causes of liver function abnormalities in present-day expectant mothers.

In this issue of Digestive Diseases and Sciences, Duraiswamy et al. [5] likewise re-examine this clinical axiom, hypothesizing that acute viral hepatitis is no longer the leading cause of hyperbilirubinemia in expectant mothers in the USA. The authors investigated causes of hyperbilirubinemia in a relatively homogenous contemporary population in Dallas, Texas. This single-institution retrospective review identified 397 deliveries from mothers with total bilirubin levels $>1.2 \mathrm{mg} / \mathrm{dl}$ obtained from prenatal care testing. A thorough chart review of cases from 2005 to 2011 was used to determine the etiology of the elevated plasma bilirubin concentrations. In indeterminate cases, an experienced hepatologist and obstetrician applied standard definitions of the relevant pregnancy- and non-pregnancyrelated causes of hyperbilirubinemia delineated in the study in order to best ascertain etiology.

Among the total number of deliveries during the study $(80,408)$, the rate of hyperbilirubinemia $(0.5 \%)$ was consistent with data previously reported in the literature [1]. Greater than $75 \%$ of the maternal population in this study - both hyperbilirubinemic and control groups-was Hispanic. Gallstone-related disease was the single most common cause of hyperbilirubinemia (25\%). Pre-eclampsia/eclampsia and hemolysis, elevated liver enzymes, and low platelet count (HELLP) syndrome (24\%) and intrahepatic cholestasis of pregnancy $(13 \%)$ were the next most common etiologies. Of the four pregnant women with "acute hepatitis" only one mother had acute hepatitis B and none were diagnosed with hepatitis A or E. Patients with acute hepatitis had higher plasma concentrations of aspartate and alanine aminotransferases (AST and ALT, 
respectively) and bilirubin. Hyperemesis gravidarum was the most common cause of hyperbilirubinemia in the first trimester (43\%), gallstone-related disease was most common in the second trimester (31\%), and pregnancy-related diseases accounted for the majority of third trimester etiologies (30\%). Pre-term delivery, fetal malformation, and death were more common in women with hyperbilirubinemia than in controls (44 vs. $11 \%, p<0.001$ ); this difference persisted after multivariate analysis [OR 2.42 (95\% CI 1.43-4.10), $p<0.01]$. Adverse fetal outcomes correlated with the severity of the underlying etiology.

In the global context, this study highlights an important paradigm shift in the cause of abnormal liver tests in pregnancy following widespread implementation of hepatitis $A$ and $B$ vaccination. While the authors identify gallstone-related disease as the most common cause of hyperbilirubinemia, the preponderance of a Hispanic population in this study raises the question of whether these findings would apply to a broader-based US population. Pregnancy-related disease, the second most common etiology in the current study, is by far the most common cause in Europe and Asia [6-8]. Further, gallstone-related disease is much less frequent outside of the USA. Additional studies are needed to understand whether these findings reflect the US population at large or are specific to a Hispanic population in Texas.

Acute viral hepatitis was infrequent, likely a reflection of vaccination trends. Likewise, no patients in a European cohort were diagnosed with acute viral hepatitis [6]. Although a Korean cohort had the highest percentage of acute hepatitis B infection (12\%), it was still less common than pregnancy-related liver disease [9]. Patients with acute hepatitis experienced the highest initial and peak levels of total bilirubin, AST, and ALT. Significantly elevated liver tests should raise the clinical suspicion for an infectious or ischemic etiology. Adverse fetal outcomes were higher in the study group than controls and were more prevalent in severe diseases including obstetric and non-obstetric underlying etiologies.

Duraiswamy et al. highlight an important paradigm shift in the approach to abnormal liver tests in the pregnant patient. The authors convincingly report that the incidence of acute hepatitis B in the jaundiced pregnant patient has decreased significantly-from 40 to $0.2 \%$ - since the landmark study from the same institution in 1977. This study also highlights the importance of timing of presentation of abnormal liver tests in aiding the determination of etiology. Additional studies are needed to validate these results in other, perhaps more diverse, US populations. The authors substantiate that the first step in evaluating a woman with abnormal liver tests in any stage of pregnancy should be no different than in a non-pregnant patient. It is important to note, however, that patients with gallstone disease tended to be Hispanic and obese, possibly reflecting a larger trend throughout the USA, although additional studies with a more representative US sample are needed for confirmation.

\section{Key Messages}

- Early identification of abnormal liver tests in the pregnant patient and appropriate diagnosis is essential for clinical decision-making.

- The incidence of acute viral hepatitis in pregnancy has declined precipitously worldwide.

- Obstetric-related liver diseases are the most common causes of abnormal liver tests in the pregnant patient globally. This predominantly Hispanic population more frequently experienced gallstone-related disease.

- Clinical suspicion should account for patient-specific geographic and ethnic risk profile.

- Further studies are needed to better understand the prevalence of gallstone-related disease in a representative sample of the US population.

\section{Compliance with ethical standards}

Conflict of interest The authors declare that they have no conflict of interest.

\section{References}

1. Tran TT, Ahn J, Reau NS. ACG clinical guideline: liver disease and pregnancy. Am J Gastroenterol. 2016;111:176-194.

2. Bennett NM, Forbes JA, Lucas CR, Kucers A. Infective hepatitis and pregnancy: analysis of liver function test results. Med J Aust. 1967;2:974-976.

3. D'Cruz IA, Balani SG, Iyer LS. Infectious hepatitis and pregnancy. Obstet Gynecol. 1968;31:449-455.

4. Adams RH, Cobes B. Viral hepatitis during pregnancy. JAMA. 1965;192:195-198.

5. Duraiswamy S, Sheffield JS, Mcintire D, et al. Updated etiology and significance of elevated bilirubin during pregnancy: changes parallel shift in demographics and vaccination status. Dig Dis Sci. (Epub ahead of print). doi:10.1007/s10620-016-4282-3.

6. Ch'ng CL, Morgan M, Hainsworth I, Kingham JG. Prospective study of liver dysfunction in pregnancy in Southwest Wales. Gut. 2002;51:876-880.

7. Mishra N, Mishra VN, Thakur P. Study of abnormal liver function test during pregnancy in a tertiary care hospital in Chhattisgarh. $J$ Obstet Gynaecol India. 2016;66:129-135.

8. Wong HY, Tan JYL, Lim CC. Abnormal liver function tests in the symptomatic pregnant patient: the local experience in Singapore. Ann Acad Med Singap. 2004;33:204-208.

9. Kim JH, Kwon CI, Ko EH, et al. Abnormal liver function tests in pregnancy: a single institution experience. Korean J Gastroenterol. 2007;50:36-40. 